\title{
Casos controversiales: un estudio de la relación entre ciencia y derecho en el proceso judicial
}

\author{
Controversial Cases: A Study of Relations between Science \\ and Law in Courts \\ Casos Polêmicos: um estudo da relação entre ciência e direito \\ no processo judicial
}

\author{
Gonzalo LuCiano Bailo* \\ NORMA ELIZABETH LEVRAND**
}

FECHA DE RECEPCIÓN: 20 DE ENERO DE 2017. FECHA DE APROBACIÓN: 17 DE OCTUBRE DE 2017

DOI: http://dx.doi.org/10.12804/revistas.urosario.edu.co/sociojuridicos/a.5341

Para citar este artículo: Bailo, G. L., E Levrand, N. E. (2018). Casos controversiales: un estudio de la relación entre ciencia y derecho en el proceso judicial. Estudios Socio-Jurídicos, 20(1), 45-74. doi: http://dx.doi.org/10.12804/revistas urosario.edu.co/sociojuridicos/a.5341

\section{RESUMEN}

La participación de expertos y la utilización de prueba científica en procesos judiciales se han incrementado, articulando prácticas e imaginarios del sistema jurídico y del tecnocientífico. En estos procesos judiciales, puede distinguirse un grupo de 'casos controversiales', que problematizan la autoridad de jueces y expertos y renegocian los significados de conceptos tradicionales, como el de 'neutralidad judicial' e 'imparcialidad e independencia del experto'. A fin de contribuir en la caracterización de estos casos controversiales, en este trabajo se propone una matriz de análisis diseñada a partir de conceptos provenientes del campo de los estudios sociales de la ciencia y la tecnología (ESCT) y de la dogmática jurídica. Esta matriz se construye a partir de una dimensión formal/estructural, una material/funcional y una institucional/decisional. Estas dimensiones se complementan para ofrecer una perspectiva que permita caracterizar los casos controversiales y diferenciarlos

* Abogado, becario doctoral Conicet (Argentina). Docente simple de Derecho Civil II (Obligaciones) y de Derecho de Daños en FCJS-UNL. Desarrolla su investigación en propiedad intelectual de plantas y semillas y en regulación de las nanotecnologías. Lugar de trabajo: Centro de Investigaciones FCJS-UNL. Correo electrónico: gbailo@outlook.com. Orcid: 0000-0002-7395-2630.

** Abogada, becaria posdoctoral Conicet (Argentina). Docente adjunta del Seminario de Relaciones Laborales en la Facultad de Ciencias de la Gestión (Uader), jefa de Trabajos Prácticos de Ciencia, Tecnología y Sociedad en la Facultad de Ingeniería y Ciencias Hídricas (UNL). Desarrolla su investigación sobre la tutela jurídica del patrimonio cultural y trabaja sobre el vínculo naturaleza-sociedad en la regulación jurídica. Correo electrónico: normalevrand@gmail.com. Orcid: 0000-0002-1396-3688. 
de otros casos complejos. A modo ilustrativo, se incluyeron algunos casos ambientales de la jurisprudencia argentina.

Palabras clave: casos controversiales, prueba científica, estudios sociales de la ciencia y la tecnología, casos ambientales.

\begin{abstract}
There has been an increase in the use of scientific evidence and expert participation in several court cases, by multiple articulations between the practices and imaginaries from legal and techno-scientific systems. In these cases, a group of 'controversial cases' can be distinguished, which problematize the authority of judges and experts, and renegotiate the meanings of traditional concepts such as 'judicial neutrality' and 'expert impartiality and independence'. In order to contribute characterizing such controversial cases, in this paper it is proposed an analysis matrix designed with concepts from Social Science and Technology Studies (STS) and legal studies. This matrix is built from three dimensions: formal/structural, material/ functional and institutional/decisional. These complementary dimensions offer a perspective that allows characterize controversial cases and difference them from other types of complex cases. As example, it is included some environmental cases selected from Argentinian courts.
\end{abstract}

Key words: Controversial cases, scientific evidence, social studies of science and technology, environmental cases.

\title{
RESUMO
}

A participação de expertos e a utilização de prova científica em processos judiciais se têm incrementado, articulando práticas e imaginários do sistema jurídico e do tecnocientífico. Nestes processos judiciais, pode distinguir-se um grupo de "casos polêmicos", que problematizam a autoridade de juízes e expertos e renegociam os significados de conceitos tradicionais como o de "neutralidade judicial" e "imparcialidade e independência do experto". Com o fim de contribuir na caracterização destes casos polêmicos, neste trabalho propõe-se uma matriz de análise criada a partir de conceitos provenientes do capo dos Estudos Sociais da Ciência e a Tecnologia (ESCT) e da dogmática jurídica. Esta matriz se constrói a partir de uma dimensão formal/estrutural, uma material/funcional e uma institucional/decisional. Estas dimensões se complementam para oferecer uma perspectiva que permita caracterizar os casos polêmicos e diferenciá-los de outros casos complexos. A modo ilustrativo, se incluíram alguns casos ambientais da jurisprudência argentina.

Palavras- chave: casos polêmicos, prova científica, estudos sociais da ciência e a tecnologia, casos ambientais. 


\section{Introducción: ciencia y derecho en el proceso judicial}

Vivimos en una sociedad en la cual el conocimiento se ha convertido en una habilidad indispensable para actuar socialmente, para estructurar la realidad (Stehr, 2013, p. 59). Las prácticas científicas y tecnológicas innovadoras modifican, día a día, nuestras condiciones de existencia. Al mismo tiempo, el conocimiento científico se ha tornado cuestionable, incierto, por lo que la comprensión de su utilización en ámbitos extracientíficos exige capacidades cognitivas que no poseemos.

En esta coyuntura, el conocimiento, como capacidad de actuar, se manifiesta en circunstancias que permiten un margen de disposición, o una necesidad de decisión. En el proceso judicial, los operadores jurídicos trabajan, cada vez con más frecuencia, con casos en los que la producción de prueba científica y la participación de expertos resulta determinante para los resultados del litigio. ${ }^{1}$ Voces y prácticas autorizadas de distintas disciplinas se incorporan al expediente y se convierten en eficaces recursos para la gestión estratégica de los intereses en el ámbito judicial.

Esta apertura del campo jurídico a las prácticas tecnocientíficas ha sido objeto de análisis particular en las ciencias sociales. Desde los años treinta, un conjunto de estudios sobre la producción del conocimiento científico ha puesto en crisis la concepción autónoma de este. La sociología de la ciencia y los estudios sociales de la ciencia y la tecnología (en adelante ESCT) se posicionaron como un campo interdisciplinario y multitemático, cuyo objeto principal de investigación es la organización, producción y circulación del conocimiento científico. Desde los años sesenta, estos estudios se radicalizaron, a partir de corrientes constructivistas, relativistas, teorías de redes de actor, entre otras. Todas estas corrientes coincidieron en cuanto a que las prácticas científicas se determinan socialmente y tienen un carácter contingente, relativista y local (Shinn, 1999, p. 15).

El análisis particular de las prácticas tecnocientíficas en el derecho, desde estos enfoques, fue desarrollado durante los años ochenta.

1. En estos casos, la utilización de conocimientos científicos se presenta como un elemento esencial para arribar a una decisión judicial. Sin embargo, la complejidad y penetración de estos conocimientos en múltiples aspectos de la vida fragmentan la capacidad de actuar de los operadores jurídicos, y exigen, para comprender su significado, la actuación de un sujeto especial: el experto (Jasanoff, 2005a, p. 53). 
A partir del estudio de controversias concretas, los ESCT pusieron de manifiesto una construcción dialéctica y problemática de la verdad en la decisión judicial.

Jasanoff y Nelkin consideran que los procesos judiciales han tenido que lidiar con dos tipos de casos controversiales: aquellos en los que los avances científicos han motivado debates éticos de distinto tipo y aquellos que involucran riesgos sociales que se perciben insuficientemente mitigados por parte de las autoridades (Jasanoff y Nelkin, 1982, p. 268).

Estas controversias ponen en contacto imaginarios que provienen de las prácticas del sistema jurídico (justicia en el caso concreto, neutralidad judicial) e imaginarios que resultan de prácticas tecnocientíficas (confianza en el conocimiento científico, imparcialidad del experto). Para algunos juristas, el incremento del uso judicial de los conocimientos científicos reduce proporcionalmente el uso del sentido común, y aumenta, por lo tanto, el grado de certeza y de posibilidad de control de la información de la que el juez puede disponer para constatar los hechos (Taruffo et al., 2009, p. 36). Otros juristas, sin desconocer las ventajas de contar con información científica para la tarea judicial, han optado por manifestar algunos reparos respecto de la posible portabilidad de los saberes científicos a los conflictos judiciales (Denti, 1972, p. 5).

En estos casos controversiales, el grado de aceptabilidad o rechazo social que puedan alcanzar las decisiones judiciales depende en buena medida de las representaciones que circulan en el interior de una sociedad respecto de lo que es derecho y lo que es ciencia, y del modo en que jueces, expertos y partes, a través de alianzas y selecciones estratégicas, explotan esas representaciones para legitimar sus intereses.

Partiendo de la situación descripta, en este trabajo se propone caracterizar los principales aspectos jurídicos de los casos controversiales. Para abordar los aspectos conceptuales, se utilizaron materiales que desarrollan distintos aspectos de la toma de decisiones judiciales en contextos de controversia científica, provenientes del campo de los ESCT y de la dogmática jurídica procesal. Para ilustrar los aspectos prácticos, se seleccionaron algunos casos ambientales argentinos que dan cuenta del estado actual de este tipo de litigios en dicho país. La selección fue realizada con base en la conjunción de: la participación procesal de los expertos de distintos 
campos científicos, el manejo procesal de la controversia científica y el sustrato ambiental del pleito.

La caracterización de los casos controversiales se efectúa a partir de la construcción de una matriz de análisis organizada en tres dimensiones. En la dimensión formal/estructural, se desarrolla el problema de la utilización de la prueba y la experticia científica en el proceso. En la dimensión material/funcional, se recupera el análisis de las tensiones que se producen en el proceso entre valores propios de las prácticas legales y valores propios de las prácticas tecnocientíficas. Finalmente, en la dimensión institucional/ decisional, se aborda el problema de la posible aceptación o rechazo de la respuesta judicial en relación con otros ámbitos de participación. Estas dimensiones son desarrolladas en los tres siguientes títulos del trabajo, presentándose antes de las conclusiones una tabla que las sintetiza. El diseño de los títulos y subtítulos tiene el propósito de adelantarle al lector la caracterización de los casos controversiales que este trabajo propone.

En este sentido, el objetivo del texto es que la matriz ofrecida en la tabla 1 permita reconocer casos controversiales, distinguirlos de otros casos complejos y delinear tres dimensiones que posibiliten examinarlos a partir de la convergencia teórica propuesta.

\section{En su aspecto formal los casos controversiales importan}

\subsection{Un ejercicio de coproducción de neutralidad}

Una mirada crítica de la utilización de la ciencia en los tribunales impide asumir que las prácticas y métodos de las comunidades científicas puedan trasladarse sin más al ámbito del proceso. El derecho tiene sus propias necesidades institucionales y restricciones, $\mathrm{y}$, a diferencia de lo que sucede en la comunidad científica, el juez no puede fallar en el caso concreto sin considerar la admisibilidad y confiabilidad de la evidencia científica que se incorpora al expediente (Jasanoff, 2006, p. 329).

La ciencia en el proceso no conlleva hechos puros o verdades reveladas, sino que ingresa bajo una forma particular, que el derecho considera pertinente, esto es, como prueba. La prueba científica se ajustará, 
epistemológicamente, a los criterios elaborados desde el derecho, y dejará de lado otros que pertenecen a los debates científicos. ${ }^{2}$ Ejemplo de ellos son: la relevancia, la oportunidad y el test de admisibilidad.

Estos criterios jurídicos apuntan a satisfacer una de las necesidades institucionales del derecho, la creación de una 'verdad' dentro de los procesos judiciales. Esta verdad se co-construye entre operadores jurídicos y científicos, a partir de la determinación y lectura conjunta de la plataforma fáctica. El juez, para cumplir adecuadamente sus funciones, debe apoyarse en la actuación de otros sujetos procesales que, como el perito o el experto, se presuponen confiables por su idoneidad técnica e imparcialidad. Ellos son tratados sugestivamente por la legislación como una especie del género "auxiliares de justicia". ${ }^{3} \mathrm{El}$ perito es un auxiliar del conocimiento del juez para la solución del pleito como lo puede ser el testigo, el documento o el informe.

El juez y el experto, pese a ser operadores de distintos sistemas sociales, actúan en el proceso como terceros no interesados en el desenlace del conflicto. Esta co-construcción de neutralidad procesal, esta empresa común de saberes científicos y jurídicos, presupone -o está obligada a presuponer- que los hechos científicos que se importan al proceso son incontrovertibles (Jasanoff, 2006, p. 332).

En el estudio de esta relación de auxilio y complementariedad entre el sistema jurídico y el científico, Jasanoff pone de manifiesto una serie de similitudes y diferencias entre ambos. En primer lugar, tanto la ciencia como el derecho generan confianza, es decir, se espera que los resultados de ambos sistemas sean imparciales y desinteresados; en segundo lugar, ambos son soportes privilegiados para legitimar decisiones públicas, es

2 Así, en uno de los casos estudiados, la controversia giraba en torno a la prueba de la contaminación por filtración de hidrocarburos en un terreno aledaño a la unidad extractiva, presentada por la parte demandada. En él se dijo que "la prueba pericial, que es prueba producida por terceros, tiene un modo específico de control por los litigantes y un procedimiento adecuado para que se verifique el contradictorio, asegurando el derecho de defensa de los contendientes [...]. Si el mismo no se respeta [...] se viola aquel principio del debido proceso y la buena fe del otro litigante que tuvo derecho a tener por consentidas las conclusiones del experto que no fueron cuestionadas" (Gioachini, Adolfo vs. YPF S.A. s. Daños y perjuicios. Tercera Cámara de Apelaciones en lo Civil, Comercial, Minas, de Paz y Tributario, Mendoza. 12/08/2015).

3 Se ha dicho que "[...] nunca el experto en las ciencias duras sustituirá al juez, sino que aquél será siempre un auxiliar porque le es útil, lo apoya en su actividad de formar su convicción a través de los conocimientos científicos [...]" (Carbone, 2012, p. 403). 
decir, son fuentes de autoridad y conocimiento; finalmente, ambos buscan traducir, representar del modo más fiel posible la realidad - la ciencia a partir de los instrumentos y tecnologías disponibles, y el derecho a través de sus reglas de evidencia- (Jasanoff, 2005a, p. 49).

No obstante ello, el derecho también presenta especificidades que limitan el auxilio que este puede solicitar al conocimiento científico. En primer lugar, el derecho está llamado a resolver casos concretos, y ante un conflicto los tribunales deben aplicar criterios jurídicos para admitir y valorar la prueba científica. En segundo lugar, estos criterios de legitimación y control de la evidencia científica tienen un ámbito particular y restringido de actuación: el proceso judicial. Por último, los jueces tienen la obligación de resolver los casos aun en situaciones de incertidumbre o insuficiencia del conocimiento científico (Jasanoff, 2005a, pp. 49-51).

Estas propiedades distintivas del sistema jurídico explican, en buena manera, que parte de las evidencias disponibles en materia de controversias científicas (toxicidad de ciertos químicos, impactos de un emplazamiento industrial determinado, efectos nocivos de ciertos productos elaborados, etc.) no provengan de una ciencia pre/extra/litigio, sino que, por el contrario, resulten de las particulares formas pos/intra/litigio que puedan asumir los debates en un expediente judicial.

\subsection{Que transita por los cauces del proceso judicial}

La incorporación de prueba científica al proceso plantea al juzgador el problema de la vinculación de los materiales científicos con su decisión (Carbone, 2012, p. 390), y el consecuente riesgo de elevar los resultados obtenidos a la categoría de prueba legal (Gozaíni, 2015, p. 172). Por un lado, la preparación de los operadores jurídicos resulta fundamental para comprender y analizar una prueba que se presenta altamente especializada y técnica. ${ }^{4}$ Pero, por otro lado, los jueces en el proceso no están llamados a resolver conflictos científicos o técnicos, sino casos concretos, en los que las cuestiones valorativas, los conocimientos que constan fuera del

4 De este modo se ha indicado que los conflictos que pueden generar el lenguaje, tecnicismo, alta especialización y valoración de la prueba científica no deben ir en desmedro del legítimo derecho de defensa en juicio de los justiciables (Despacho de la Comisión de Prueba Científica, XXIV Congreso Nacional de Derecho Procesal, 2007). 
proceso y las máximas de experiencia que los tribunales sedimentan en sus precedentes también pueden asumir un papel determinante.

El derecho procesal no dispone de regulación específica para la prueba científica. En Argentina, la doctrina mayoritaria considera la prueba científica como una especie dentro del género de las pruebas periciales. ${ }^{5}$ Sin embargo, algunos autores han formulado ciertos reparos a esta propuesta, ${ }^{6}$ especialmente cuando existen particularidades de la prueba científica que impiden mantener la singularidad de la técnica pericial (Gozaíni, 2015, p. 171). También se han marcado los atributos de la información científica cuando se presenta bajo otros cauces, principalmente como especie de prueba documental (Peyrano, 2007, p. 109) o testimonial (Gozaíni, 2015, p. 172).

Es posible reconocer al menos dos vías principales para incorporar la información científica al proceso. Por un lado, se admite la prueba pericial cuando "la apreciación de los hechos controvertidos requiera conocimientos especiales en alguna ciencia, arte, industria o actividad técnica especializada" (Código Procesal Civil y Comercial de la Nación Argentina, CPCyCN, artículo 457; la cursiva es nuestra); y, por otro lado, se acoge la producción de prueba científica a partir de la regulación de los medios de prueba no previstos. ${ }^{7}$

Otras vías previstas por la regulación, que son utilizadas con menor frecuencia, son los exámenes científicos ${ }^{8}$ o consultas científicas o técnicas. ${ }^{9}$ Estas últimas, que pueden dirigirse a universidades, academias o instituciones científicas o técnicas, se denominan 'pericial informativa' o 'pericial colegiada'. Se entiende que, si recaen sobre el marco general

5 Se han señalado como características distintivas de su especie, entre otros aspectos: su método, su elaboración y la especialidad del experto que la lleva a cabo (Despacho de la Comisión de Prueba Científica, XXIV Congreso Nacional de Derecho Procesal, 2007).

6 Por ejemplo, Gozaíni ha expresado que "si la prueba científica no se aísla de la prueba pericial, puede ocurrir que la especialidad del medio quede atrapado en las redes de la experticia, caracterizada con la emisión de un dictamen simple de evaluación, o de conclusiones elaboradas después de la celebración" (2012, p. 173).

7 Expresa el СРСуC que "los medios de prueba no previstos se diligenciarán aplicando por analogía las disposiciones de los que sean semejantes o, en su defecto, en la forma que establezca el juez" (art. 378).

8 Estos incluyen aquellos dispositivos y elementos de producción de conocimiento que permitan "el mejor esclarecimiento de los hechos controvertidos" (CPCyCN, art. 475).

9 Cf. CPCyCN, art. 476. 
del debate y no sobre el litigio concreto, en caso de divergencias pueden imponerse por sobre la pericial común. "Es que la pericial contempla la causa concreta y, en general, la 'consulta científica' se formaliza más o menos abstractamente y de manera desasida de las circunstancias del caso" (Peyrano, 2012, p. 57).

Teniendo en cuenta estas circunstancias, en casos controversiales - donde habitualmente se debaten cuestiones técnicas de gran complejidadel juez podría apartarse, o bien complementar las periciales comunes a partir de las opiniones vertidas en una consulta científica. ${ }^{10}$

La interpretación de la prueba científica, por su parte, exige cierta planificación estratégica por el juez, no solo porque tiene que ordenar la producción de la prueba, sino porque luego tendrá que valorarla (Rojas, 2012, p. 265). ${ }^{11}$ En una sociedad democrática no es dable renunciar al control de lo que sucede en el proceso, "esto, y no otra cosa significa la libertad de valoración que se atribuye al juzgador respecto a la prueba pericial; y es a través de la motivación de sus propias decisiones [...] como el juzgador debe rendir cuentas [...] del control que ha realizado sobre la labor del perito" (Denti, 1972, p. 20).

A modo de ejemplo, cuando el juez debió valorar, desde las reglas del debido proceso, la posible colisión entre estudios producidos fuera del proceso y prueba científica rendida dentro del proceso, indicó: "Las cuestiones científicas corresponden ser zanjadas en el trámite de las pruebas periciales. Los estudios que la demandada acompañó con su contestación de demanda no pudieron tener el control de la contraparte, es decir de la actora, por lo que no es razonable ni aceptable en términos de debido proceso (art. 18, Constitución Nacional) que puedan predominar por sobre las experticias". ${ }^{12}$

10 Para algunos autores, el hecho de presentarse desasida de las circunstancias del caso y provenir de instituciones de reconocida trayectoria puede erigir a la consulta científica en una pericia de mayor "autoridad" que la común (Peyrano, 2007, p. 112).

11 La eficacia probatoria del dictamen debe ser estimada por el juez de acuerdo con pautas establecidas jurídicamente, como la competencia del perito; los principios científicos o técnicos en que se funda; la concordancia de su aplicación con las reglas de la sana crítica; las observaciones formuladas por los consultores técnicos o los letrados y los demás elementos de convicción que la causa ofrezca (CPCyCN, art. 477).

12 Gioachini, Adolfo vs. YPF S.A. s. Daños y perjuicios. Tercera Cámara de Apelaciones en lo Civil, Comercial, Minas, de Paz y Tributario, Mendoza. 12/08/2015. 
Esta circunstancia nos permite distinguir, por ejemplo, la prueba científica de la pericia arbitral, dado que en esta última los peritos están autorizados para resolver cuestiones de hecho.

\subsection{Negociando cientificidad desde mundos diversos}

Dos son los principales mitos que habitualmente acompañan a la prueba científica: que siempre generan certezas absolutas; y que sus resultados son infalibles (Peyrano, 2007, p. 111). El carácter científico de la prueba no resulta de una especial legitimidad o carácter normativo atribuible en abstracto a una serie de métodos o resultados, sino del estatus privilegiado que algunas prácticas del conocimiento obtienen en el mundo social. Denti considera que el criterio de 'cientificidad' de la prueba debe determinarse en relación con la función institucional del juzgador. Se debe atender a las valoraciones que el juez realiza con sustento en conocimientos que rebasan sus saberes (Denti, 1972, p. 4). De esta manera, el autor descarta que una prueba pueda considerarse científica por los procedimientos que se utilicen para obtenerla o por los medios que se empleen para materializarla. La prueba científica es un recurso que ostenta un poder de convicción especial.

Para alcanzar este estatus, el sistema científico necesita comunicar los métodos y recursos que se utilizan en sus espacios (centros de investigación, protocolos, instrumentales, etc.) con los métodos y recursos utilizados en otros subsistemas que, como el jurídico, cuentan con sus propios espacios y procedimientos (tribunales, códigos procesales, expedientes, etc.). La prueba científica abre espacios comunes para la traducción de experticias, y representa una oportunidad para negociar la estabilidad que requiere cualquier campo científico para consolidarse y la confiabilidad que el derecho exige a los materiales que sostienen sus decisiones. ${ }^{13}$

13 Puede mencionarse como ejemplo de estas prácticas de negociación el Manual de referencia en evidencia científica que elabora el Centro Judicial Federal de Estados Unidos, dado que el comité encargado de elaborar la tercera edición del manual, además de contar con magistrados de distintos órdenes, se integró con expertos provenientes de la medicina, la ingeniería, la matemática, las ciencias del ambiente, etc. A ello también se sumó el aporte de cuatro academias nacionales en carácter de 'advisers' (National Academy of Sciences, National Academy of Engineering, Institute of Medicine, National Research Council). En este sentido, el manual pretende funcionar como una guía para trabajar con prueba científica en el proceso, pero también como fuente de información respecto de los cambios que se producen en el interior de las disciplinas científicas. 
En estos ejercicios de traducción del trabajo legal y científico, se negocia la experticia y la cientificidad de la prueba. ${ }^{14}$ Allí, los jueces inevitablemente imponen su propio entendimiento acerca de qué visión del mundo se considera autorizada o es genuinamente científica y cuál no, cuáles son los elementos aptos para lograr mayor poder de convicción y persuasión en las resultas del proceso y cuáles deben ser desestimados (Bailo et al., 2015, p. 117).

En algunos de los casos estudiados, la relación entre derecho y ciencia se construye, en los estrados judiciales, de manera pacífica "[...] se debe buscar un equilibrio entre la evolución tecnológica y el uso sustentable y sostenible de los recursos naturales, con sus consiguientes efectos en la salud humana. Con ese objetivo se fijan parámetros y límites máximos, que se van recreando, reasignando, reviendo y reconsiderando, a los nuevos conocimientos técnicos y científicos". ${ }^{\prime 5}$

En otros casos, en cambio, la relación entre derecho y ciencia se presenta problematizada desde el inicio de las actuaciones:

La ausencia de una demostración científica decisiva lleva a tener que ser prudentes [...] la existencia de una publicación [...], expresando que el herbicida utilizado para la soja transgénica, principal cultivo de Argentina, puede producir malformaciones neuronales, intestinales y cardíacas [...] pone en crisis la Resolución 350/99 de la Secretaría de Agricultura, Ganadería y Pesca [...] por la cual se consideraba al glifosato como un producto que no ofrecía peligro según los parámetros de la Organización Mundial de la Salud..$^{16}$

14 Desde la aparición del precedente Daubert v. Merrell Dow Pharmaceuticals, Inc. (509 U.S. 579, año 1993), las comunidades legales y las científicas expandieron sus ámbitos de negociación. En él, la Corte Suprema de Estados Unidos confió a los jueces el control de ciertos aspectos de los testimonios expertos, tales como el uso del razonamiento y la metodología científica.

15 Asís María Fernanda c/ Giorda, Mario Luis y otro - ordinario - otros. Cámara Quinta Civil y Comercial, Córdoba. 24/05/2007. El caso presenta un debate sobre los riesgos que podría conllevar la instalación de una antena de telefonía móvil que, según las normas vigentes, operaba bajo los estándares legalmente establecidos.

16 Del dictamen fiscal en Peralta, Viviana y otros c/ Municipalidad de San Jorge y otros s. Amparo. Juzgado de Primera Instancia de Distrito en lo Civil, Comercial y Laboral de San Jorge, Provincia de Santa Fe. 16/03/2009. En este proceso se debatieron, por un lado, los riesgos del uso de agrotóxicos en territorios semiurbanos y, relacionado con ello, la necesidad de establecer una franja territorial de protección de la salud humana más estricta. 
En los ESCT se cuestiona la premisa que postula que la delimitación entre lo que es considerado científicamente confiable y no confiable es establecida en gran parte fuera de la competencia del derecho y puede ser importada, sin problemas, dentro de procedimientos legales (Jasanoff, 1998, p. 722). ${ }^{17}$

\section{Y que en su dimensión funcional}

\subsection{Revelan profundos conflictos sociales}

Pese a esta creciente vinculación entre prácticas legales y científicas, no resulta extraño encontrar casos en los que la evidencia científica no brinde adecuado soporte a la controversia (Nelkin, 1979, 1987, 1995). Una de las principales razones de este fenómeno tiene que ver con "[...] una pérdida del respeto, sobre todo ante la autoridad estatal-administrativa y el poder de los expertos. Y esta pérdida de respeto por ningún motivo retrocede ante el conocimiento" (Stehr, 2013, p. 65). En los casos controversiales, más que el conocimiento científico, se cuestionan los valores enraizados en nuestras prácticas sociales. Esto dificulta la capacidad del juez de distinguir, en el caso concreto, los valores de los hechos, y, por ende, de identificar el conocimiento más confiable para conducir la controversia.

Los ESCT han puesto en duda que la resolución de los conflictos dependa de más investigación, mejor evidencia y mejor asesoramiento experto (Nelkin, 1987, p. 284). La producción de conocimiento científico envuelve siempre valores o intereses políticos que deben ser resueltos democráticamente. La ciencia que se hace para uso jurídico no funciona de la misma manera que la ciencia que se hace para el avance de la empresa científica y, aunque el derecho intente valerse de la autoridad

17 Según esta visión, condensada parcialmente en precedentes como Daubert, los jueces actúan como 'guardabarreras' (gatekeepers), porque tienen el poder de dejar entrar el testimonio confiable y cerrar la compuerta a voces no autorizadas. 
del conocimiento científico, nunca deja de lado su compromiso con los valores fundamentales (Jasanoff, 2006, p. 329)..$^{18}$

Esta tensión se intensifica cuando se intenta, por los cauces procesales habituales, juridificar la historia social de una controversia determinada. Normalmente, los conflictos tienen causas múltiples, se desarrollan en períodos extensos de tiempo en los que conviven numerosos cambios legales, políticos y económicos. En estos lapsos de tiempo, los interesados ensayan distintos cursos de acción según sus posibilidades, que provocan distintas respuestas por parte de actores públicos y privados. El proceso judicial, en cambio, "trabaja en tiempos relativamente cortos, con fuentes o recursos limitados y está orientado a la producción de una decisión tendencialmente definitiva sobre el específico objeto de la controversia" (Taruffo, 2005, p. 16). Entre ambas temporalidades, la ciencia se debate entre producir conocimiento anticipadamente, para ser utilizado cuando se lo requiere o la producción en el momento en que las controversias se suscitan (Nowotny, 2011, p. 338).

En algunos casos, las incertezas inherentes a la controversia logran horadar el limitado ámbito de conocimiento temporal del contradictorio. En uno de los pronunciamientos estudiados, la empresa demandada había cambiado de titularidad y modificado los criterios técnicos de explotación, no obstante se demandó la contaminación producida varios años antes, bajo otros criterios técnicos. Allí, el tribunal estimó que, durante su historia reciente, la empresa evidenció "un manejo totalmente descuidado que afectó seriamente a las aguas superficiales y al suelo", por lo que "no se puede pretender que la contaminación de años se supere en un corto plazo". ${ }^{19}$ Una de las herramientas que utiliza el tribunal para superar los inconvenientes que surgen de los cambios en el tipo de explotación, de

18 Así, se ha comenzado a admitir gradualmente que los avances tecnológicos y la comercialización de manufacturas de alto valor agregado - como las producidas con base en nanotecnologías- desafían aspectos cruciales de los regímenes regulatorios tradicionales. En estas áreas, las evaluaciones de riesgo se complejizan debido a las lagunas de información, que socavan la capacidad de las agencias estatales de advertir riesgos y realizar reformas de los marcos regulatorios existentes (Bailo, 2014, p. 112).

19 Gioachini, Adolfo vs. YPF S.A. s. Daños y perjuicios. Tercera Cámara de Apelaciones en lo Civil, Comercial, Minas, de Paz y Tributario, Mendoza. 12/08/2015. 
titularidad de la empresa, de cambios en los criterios técnicos, de sucesión de leyes en el tiempo, etc., es la de "pasivos ambientales". ${ }^{20}$

Sin embargo, no siempre las controversias logran canalizar su historia social en el proceso, en especial cuando ello puede derivar en decisiones que podrían ser tachadas de arbitrarias. Por ejemplo, en otro de los casos estudiados se ha expresado que "respecto a la no adecuación a la normativa [...] ello se debe a que la fecha de construcción de la antena es anterior a la sanción de la normativa invocada por la actora como vulnerada, no siendo aplicable al caso en cuestión -así lo indican el principio de legalidad, el principio de reserva, y el de seguridad jurídica-". ${ }^{21}$

\subsection{Que involucran valores jurídicos y científicos}

Estos casos, en los que la evidencia científica no resulta suficiente para llegar a soluciones satisfactorias, hacen públicos, ostensibles, los valores jurídicos y científicos que sedimentan nuestras prácticas sociales. ${ }^{22}$ Siguiendo los estudios de Nelkin (1995, pp. 447-449), algunas categorías de controversias entre valores pueden ser: las implicancias sociales, morales o religiosas de una teoría científica o práctica de investigación determinada; las tensiones entre valores ambientales y prioridades económicas y políticas, o entre riesgos a la salud e intereses económicos, ${ }^{23} \mathrm{y}$, finalmente, las tensiones entre expectativas individuales y metas sociales.

20 El tribunal toma como ejemplo la Ley de la Provincia de Buenos Aires 14343, que define como pasivo ambiental "al conjunto de los daños ambientales, en términos de contaminación del agua, del suelo, del aire, del deterioro de los recursos naturales y de los ecosistemas, producidos por cualquier tipo de actividad pública o privada, durante su funcionamiento ordinario o por hechos imprevistos a lo largo de su historia, que constituyan un riesgo permanente y/o potencial para la salud de la población, el ecosistema circundante y la propiedad, y que haya sido abandonado por el responsable" (art. $3^{\circ}$ ).

21 Asís María Fernanda c/ Giorda, Mario Luis y otro -ordinario- otros. Cámara Quinta Civil y Comercial, Córdoba. 24/05/2007.

22 Una de las tensiones de mayor complejidad en este terreno involucra el estatus de la cosa juzgada frente a los avances en materia de prueba científica. Se ha dicho que esta debería merecer un tratamiento legislativo específico, para que frente a casos excepcionales se habilite una revisión restringida que permita superar una situación que se hubiera transformado en intolerablemente injusta por irreal o por no haber sido juzgada adecuadamente (Despacho de la Comisión de Prueba Científica, XXIV Congreso Nacional de Derecho Procesal, 2007).

23 En estas controversias aparecen interrogantes en torno a la distribución equitativa de los riesgos, el papel que ocupan los ciudadanos en las decisiones tecnológicas y el acceso de las comunidades locales a la experticia. 
Estas disputas introducen discusiones en materia de 'derechos', y es habitual que giren en torno a la introducción de la ciencia y la tecnología en las prácticas sociales y en las dificultades que acarrea su regulación por parte de las autoridades públicas.

En muchos casos, los tribunales han concentrado mayormente su actividad en la incertidumbre técnica que en los asuntos públicos y en los problemas conceptuales que se ponen en juego (Jasanoff y Nelkin, 1982, p. 266). Sin embargo, en los casos controversiales "los opositores de cierta decisión no necesariamente tienen que presentar 'pruebas equivalentes'. En las disputas políticas y jurídicas muchas veces es suficiente plantear preguntas críticas ante los expertos para así relativizar o incluso socavar su monopolio de experticia" (Stehr, 2013, p. 65). En uno de los casos estudiados, el tribunal consideró que "la mayor compañía petrolera del país [...] se limitó a traer estudios atribuidos a equipos científicos que en juicio no fueron verificados y a tratar de obtener un beneficio procesal de la lectura sesgada de la pericia del ingeniero en petróleo" ${ }^{24}$ En estos casos, de complejidad técnica, debate científico y partes desiguales, se ha recurrido a la teoría de la colaboración probatoria, también llamada de las cargas dinámicas.

\subsection{Difíciles de traducir con las reglas del contradictorio}

Sin embargo, en estas controversias sucede algo paradójico, los cuestionamientos a la experticia y las demandas de mayor participación de los afectados también exigen una gran dependencia de la experticia científica (Nelkin, 1987, p. 288). Ello hace que, en el específico ámbito procesal, los debates acerca de la admisibilidad e interpretación de las metodologías y evidencias científicas se tornen más complejos. Se suele pensar que la resolución de la incerteza científica y técnica se traducirá automáticamente en una resolución de las tensiones ético-sociales. No obstante, en las controversias resultan cruciales las inseguridades y discrepancias que puedan mostrar los expertos, más que los hechos que desatan el conflicto, pues sirven igualmente "como estímulo y justificación para

24 Gioachini, Adolfo vs. YPF S.A. s. Daños y perjuicios. Tercera Cámara de Apelaciones en lo Civil, Comercial, Minas, de Paz y Tributario, Mendoza. 12/08/2015. 
las actividades políticas de los afectados (y también para las decisiones finalmente tomadas)" (Stehr, 2013, p. 65). ${ }^{25}$

En los ESCT se recurre a la traducción como dispositivo que vincula la producción de conocimiento científico a la producción de verdades jurídicas. Las traducciones permiten que los hechos externos al mundo jurídico ingresen al ámbito procesal, de modo que puedan ser representados y contestados en el proceso. En este sentido, la visualización, no menos que la verbalización, es una de las técnicas a través de la cual la evidencia científica logra credibilidad y gana, para propósitos de toma de decisiones legales, el estatus de un hecho (Jasanoff, 1998, p. 716). ${ }^{26}$

No resulta sencillo, con las reglas del contradictorio, garantizar a los interesados igualdad de condiciones (paridad de armas) a la hora de objetar traducciones verbales o visuales expertas, dado que el caudal de recursos, las redes institucionales y las fuentes de información que se necesitan para construirlas por lo general permanecen fuera de los márgenes procesales. En uno de los casos analizados, se evidencia que la traducción visual de la información científica en el proceso puede convertirse en un elemento determinante para imponerse sobre otros materiales, especialmente cuando se trata de persuadir al juzgador respecto de hipótesis con contenidos técnicos. ${ }^{27}$

25 La necesidad de informarse sobre las incertidumbres de los expertos y las insuficiencias del conocimiento científico también deriva en un cuestionamiento respecto de la aptitud de las reglas del contradictorio para acreditar, de la manera más fiel posible, tales extremos. Se ha dicho que "si la ciencia viene empujando nuevos estándares será necesario revisar el modelo de la apreciación racional o de la valoración objetiva [de la prueba]" (Gozaíni, 2015, p. 17).

26 Además del lenguaje, como primer modo de traducción, en los casos controversiales es usual que los expertos se valgan de las traducciones visuales. Cuando una controversia se ventila en los estrados judiciales, los expertos deben realizar un esfuerzo extra para lograr que su versión logre persuadir a jueces y partes. La sala de juicio se convierte en un teatro y el proceso, en un ejercicio performativo, en el que los hechos son narrados y presentados con la intención de ganar credibilidad.

27 En el ejemplo, el tribunal se encontró con pericias que atribuían el origen de la contaminación del suelo a causas diferentes: "El perito ingeniero de petróleos señala que el pozo de agua de la actora se encuentra aguas arriba de la pileta Ue-72, [...], por lo que resulta imposible que el pozo de agua dulce haya absorbido o absorba contaminantes de una inexistente fuente peligrosa ubicada aguas abajo. Ahora bien, el perito agrónomo y la Sra. jueza ya se refirieron al fenómeno del cono de depresión y la curva de abatimiento que se produce cuando una bomba de agua succiona. Todo está graficado por el perito a fs. 882 vta. y es esclarecedor" (Gioachini, Adolfo vs. YPF S.A. s. Daños y perjuicios. Tercera Cámara de Apelaciones en lo Civil, Comercial, Minas, de Paz y Tributario, Mendoza. 12/08/2015). 
Jasanoff (1998, p. 728) considera que se requieren ciertas restricciones en la deconstrucción de la visión experta y entiende que la estandarización ofrece, a los laboratorios de pruebas y a las instituciones de aplicación de la ley, un atractivo camino de salida para los dilemas que se plantean en la sala de juicio. Una vez que las técnicas de visualización están estandarizadas, la violación del estándar puede ser condenada en términos normativos, igualmente persuasivos para expertos y legos. Los estándares pueden ser vistos como un dispositivo de traducción que torna accesibles a los legos los juicios expertos sobre prácticas técnicas (Bailo et al., 2015, p. 117).

El debate en materia de estándares técnicos es una fuente de controversias recurrente en el campo ambiental. En uno de los casos estudiados, los jueces estimaron que, por más que el perito ingeniero en petróleos "exprese que la demandada ya cumple toda la normativa nacional y provincial en materia ambiental [...], ello no exime de responsabilidad civil". Primero, porque "está demostrado incumplimiento pretérito". Segundo, "porque aun cumpliendo luego con los estándares ambientales puede haber igual daño. Puede haber irresponsabilidad administrativa en materia ambiental, pero ello es independiente de la responsabilidad civil que pueda darse y así está definido por la Ley General del Ambiente". ${ }^{28}$

Distinto es el razonamiento que se deriva de otro de los casos estudiados, en el que se pretendía hacer funcionar la tutela precautoria en materia de radiaciones no ionizantes. En este caso, el cumplimiento de los estándares vigentes operó como elemento de convicción respecto de la inocuidad de las radiaciones no ionizantes. "Manifiesta el perito técnico que cumplimentando con las normas vigentes en cuanto a emplazamientos de las estaciones base para telefonía celular como para SCP y su nivel de potencia irradiada, éstas no resultarían nocivas para la comunidad". En el caso, "las antenas especificadas en la demanda cumplen con la reglamentación nacional emitida por la Secretaría de Comunicaciones y por la Comisión Nacional de Comunicaciones [...] Por tanto [...] el funcionamiento de la antena cumple con las medidas de seguridad contenidas en la normativa aplicable al caso, presumiéndose

28 Ibid. 
inocuo para la salud -más allá de la falta de certeza científica absoluta en cuanto a efectos para el organismo humano-". ${ }^{29}$

\section{Trasladando tensiones a instancias decisorias}

\subsection{Que demandan nuevos arreglos institucionales}

Desde los ESCT, Nelkin trató de explicar cómo las controversias acercan a los científicos al terreno de la cultura política, y de demostrar cómo la democratización del poder político problematiza la autoridad del experto en los asuntos públicos (1979, 1987, 1995). Para esta autora, la ciencia es un recurso político, que funciona como fuente de racionalidad y base de consenso en un amplio espectro de asuntos públicos. No obstante ello, así como puede legitimar decisiones, puede ser también un instrumento para cuestionarlas (Nelkin, 1987, p. 283). De esta manera, a medida que ciertas tecnologías se perciben controversiales, los propios científicos, cuya experticia es la base de las decisiones técnicas, se ven envueltos también en discusiones públicas (Nelkin, 1975, p. 35).

Este fenómeno problematiza los modelos vigentes de participación experta en los asuntos públicos y, en el caso que nos ocupa, de participación experta en el proceso judicial. ${ }^{30}$ Sin embargo, para abordar el problema del valor del juicio experto en áreas controversiales, de gran dinámica económica y social, los ordenamientos se han integrado con normas especiales. El experto, en estos órganos especiales, se piensa como un enlace entre el juzgador y la realidad social en transformación (Denti, 1972, p. 15).

Se crean instituciones donde los expertos, como agentes políticamente neutros, tienen un poder significativo para operar respecto de ciertos problemas sociales. Estas instituciones, como los comités de ética, consejos

29 Asís María Fernanda c/ Giorda, Mario Luis y otro - ordinario - otros. Cámara Quinta Civil y Comercial, Córdoba. 24/05/2007.

30 Para autores como Denti, históricamente la participación de los peritos en el proceso ha asumido tres modalidades: a) integración de colegios judiciales con expertos designados de modo continuativo o elegidos de acuerdo con la naturaleza de la controversia; b) designación del experto por el juzgador, con funciones consultivas relacionadas a la exigencia del proceso; c) presentación de peritos por obra de las partes (1972, p. 11). 
de expertos o, en casos ambientales, los comités de seguimiento ambientales, conforman un 'filtro' respecto a los cuestionamientos posibles a la producción científica como también a la toma de decisiones en cada caso. Se apoyan en la autoridad científica de sus miembros, y vuelven colectiva (pero limitada a sus miembros) aquellas decisiones políticas relativas a la inserción de innovaciones científicas y tecnológicas en la sociedad.

Por ejemplo, en otro de los casos estudiados, la empresa demandada intentó rebatir los informes del CIMA (Centro de Investigaciones del Medio Ambiente, Departamento de Química, Facultad de Ciencias Exactas, Universidad Nacional de La Plata, Argentina) con pericias alternativas, dada la controversia que existía respecto de la forma de medir la contaminación ambiental en relación con los estándares tolerados por la legislación. ${ }^{31} \mathrm{El}$ tribunal se apoyó en los informes del CIMA, que, en sucesivas mediciones, verificaron la existencia de daño ambiental por acción contaminante del aire y rebatieron las metodologías de los peritos de parte. ${ }^{32}$

No obstante ello, la incorporación del asesoramiento científico en la regulación es todavía problemática. El programa de la modernidad, que mantenía a la ciencia aislada de la política, hoy se encuentra sensiblemente controvertido (Miller, 2004, p. 46). Las instituciones, a través de rutinas administrativas estables, permiten diseñar respuestas para problemas novedosos, ensayando repertorios, optando por formas de experticia y procesos de investigación, por métodos que aseguren credibilidad y por mecanismos para ventilar y gestionar el disenso (Jasanoff, 2004, p. 40). En el derecho, las instituciones son requeridas para interpretar evidencia, sancionar leyes, estandarizar métodos o diseminar conocimiento.

En el ámbito específico del proceso judicial, las instituciones han funcionado como 'delegados' del juez a fin de controlar el cumplimiento de las sentencias. En Argentina esta actuación experta se ha cristalizado

31 Los expertos de la demandada pretendían distinguir entre normas de emisión y normas de calidad del aire. A su entender, las emisiones de las chimeneas de la empresa no son lo que efectivamente respiran las personas al nivel del suelo, por lo que en el caso no había daño ambiental.

32 "Tanto los informes del CIMA como los antecedentes remitidos por la agencia local encargada de la política ambiental dejan en claro que la empresa [...] ha infringido normas administrativas de control ambiental de emisiones gaseosas y ha superado los límites de emisiones permitidos". Del considerando 1.4.3. Mazzeo, Alicia S. y otro c/ YPF S.A. s. Cese de daño ambiental - daños y perjuicios. Cámara Federal de Apelaciones de La Plata, Sala Tercera. 12/07/2012. 
a partir del caso Mendoza, ${ }^{33}$ en el cual la Corte Suprema de Justicia de la Nación (en adelante CSIN) intimó a la Autoridad de Cuenca MatanzaRiachuelo a implementar un plan de saneamiento de la cuenca.

En consonancia con lo expuesto, Douglas Price manifiesta que en los procesos ambientales se están produciendo una serie de cambios, de carácter sistémico, entre los que se encuentran la expansión o extensión de la cosa juzgada; la flexibilización de los criterios de adopción, adquisición y valoración de la prueba; y la reordenación reflexiva de la judicatura en orden a asegurar la ejecución de sus sentencias y la efectividad de los derechos. Este autor identifica como manifestaciones propias de estos casos ambientales el apoyo en experticias provenientes de universidades nacionales, el interés en producir información confiable y accesible, la realización de audiencias públicas, la creación de autoridades administrativas con competencias especiales y la generación de modos específicos de ejecución en articulación con otras instancias judiciales e instituciones públicas (Douglas Price, 2014, p. 1).

\subsection{Que permitan la participación de colectivos más amplios}

En estas discusiones, los tribunales han sido llamados a ocupar un papel destacado a la hora de definir procedimientos para la resolución de conflictos y arbitrar controversias en aspectos sustantivos (Nelkin, 1979, p. 57; Jasanoff y Nelkin, 1982, p. 266).

Debido a los inconvenientes que pueden surgir cuando se incorpora información científica al proceso bajo los cauces procesales habituales, en algunas ocasiones los tribunales han recurrido a otros institutos. En el derecho comparado, por ejemplo, se registran antecedentes de amicus curiae en casos controversiales que involucran debates científicos (v. g., Edwards v. Aguillard, 482 U.S. 578, 1987; Universal City Studios, Inc. v. Reimerdes 111 F.Supp.2d 294 S.D.N.Y. 2000). En Argentina, la Acordada 28/2004 de la CSJN y algunas normas aisladas prevén la figura para situaciones especiales. ${ }^{34}$

33 Mendoza, Beatriz Silvia y otros c/ Estado Nacional y otros s. Daños y perjuicios (daños derivados de la contaminación ambiental del río Matanza-Riachuelo), CSJN, 20/06/2006.

34 Entre ellas la Ley 24488 de inmunidad jurisdiccional de los estados extranjeros, 25875 de procuración penitenciaria, 26827, que crea el Sistema Nacional de Prevención de la Tortura 
El amicus curiae permite la participación de terceros ajenos a las partes en asuntos de trascendencia institucional o que resulten de interés público, cuando "cuenten con una reconocida competencia sobre la cuestión debatida y que demuestren un interés inequívoco en la resolución final del caso, a fin de que ofrezcan argumentos de trascendencia para la decisión del asunto" (de los considerandos AC CSJN 28/2004). En casos controversiales, las discusiones trascienden el interés de las partes y se proyectan sobre la comunidad o ciertos sectores de ella. Así, este dispositivo permite una participación y un debate más amplio que los del contradictorio. Así mismo, el juez puede contar con información que no necesariamente proviene de las periciales, documentales o testimoniales comunes. ${ }^{35}$

En los casos ambientales se ha incluido, igualmente, un conjunto de conocimientos producidos por colectivos ciudadanos: 'mapas de la muerte', la articulación de redes en relación con experiencias similares de contaminación, o, incluso, algunos ejercicios de epidemiología popular (Berros, 2013, p. 97). En estos casos, la introducción de tales conocimientos al proceso se realiza como pruebas ofrecidas por los demandantes.

En este sentido, se ha afirmado que "en casos controvertidos científicamente, se torna muy relevante considerar las 'historias de vida', las 'experiencias', los 'saberes y conocimientos' de quienes viven cotidianamente expuestos al riesgo de que se trate". ${ }^{36}$ Se introduce, de este modo, la participación de colectivos más amplios en el proceso, no solo como demandantes, sino como productores de prueba que interpela a aquella producida científicamente.

Azuela expresa que, desde esta perspectiva, el análisis de riesgos no se concentra en los riesgos considerados en sí mismos, sino en las formas

y Otros Tratos o Penas Crueles, Inhumanos o Degradantes, y 26912, Régimen Jurídico para la Prevención y el Control del Dopaje en el Deporte.

35 De la citada reglamentación de la CSIN, se destaca que: a) los amicus curiae no son parte procesal; b) tienen participación cuando el debate involucra cuestiones de trascendencia colectiva o interés general; c) deben expresar una opinión fundada sobre el objeto del litigio, en defensa de un interés público o de una cuestión institucional relevante; d) deben tener reconocida competencia sobre la cuestión debatida y fundamentar su interés en participar en la causa; e) la incorporación de la opinión al expediente está sujeta a un test de admisibilidad por parte del tribunal; y f) las opiniones o sugerencias del amicus curiae no son vinculantes, pero pueden ser tenidas en cuenta en el pronunciamiento del tribunal.

36 Peralta, Viviana c/ Municipalidad de San Jorge y otros s. Amparo. Cámara de Apelación en lo Civil y Comercial, Sala Segunda, Santa Fe. 09/12/2009. 
de experiencia procesadas socialmente (Azuela, 2006, p. 38). Así, en otro de los casos, los jueces se han apoyado en testimonios médicos que "expresan en este mismo sentido, respecto de los problemas en las vías respiratorias de los habitantes del barrio 'Mosconi' que 'no hay una estadística hecha en la zona por organismos oficiales, pero yo creo que el número de estas dolencias es mayor al problema nacional" ${ }^{37}$

Ello demuestra las posibilidades que plantea la integración activa de los ciudadanos en diversas etapas de investigación y desarrollo científico (Jasanoff, 2011, p. 19). Ahora bien, el ámbito del proceso judicial no puede caracterizarse como tal, y la irrupción de los conocimientos populares en él requiere repensar los dispositivos de prueba y las jerarquías existentes a fin de otorgar un espacio a la epistemología cívica. ${ }^{38}$

Esta utilización de los conocimientos científicos y la 'producción' de nuevas formas de saber plantean un desafío al derecho procesal que puede designarse a partir de la conflictividad de admitir prueba científica no encuadrada como 'pericial'.

\subsection{Para hacer de la controversia un espacio de oportunidades}

En el recorrido teórico propuesto en este artículo, hemos encontrado que los casos controversiales se caracterizan por: a) la pérdida de la confianza en el conocimiento científico como custodio del interés público (Nelkin, 1995, p. 450); b) la posibilidad de reescribir las reglas de la práctica política en momentos de cambio científico y tecnológico (Nelkin, 1987, p. 284); c) la insuficiencia del modelo adversarial bipartito -actor/ demandado- (Jasanoff y Nelkin, 1982, p. 271).

Otros autores del campo de los ESCT han trabajado la relación entre ciencia y derecho en el ámbito del proceso, y parece oportuno recuperar algunos conceptos utilizados, que pueden ser repensados en relación con los casos controversiales.

37 Considerando 1.4.4. Mazzeo, Alicia S. y otro c/ YPF S.A. s. Cese de daño ambiental daños y perjuicios. Cámara Federal de Apelaciones de La Plata, Sala Tercera. 12/07/2012.

38 Este concepto refiere a aquellos modos de conocimiento público, histórico y políticamente situados, y culturalmente específicos (Jasanoff, 2005b, p. 249). Este es útil para comprender que los ciudadanos activamente integrados en los procesos de discusión sobre nuevas tecnologías y desarrollos científicos no solo 'producen' un conocimiento que puede ser utilizado en el proceso, sino que se valen de ciertos conocimientos científicamente controversiales. 
Por una parte, Hans-Heinrich Trute pone de manifiesto que, al momento de tomar decisiones, el sistema jurídico reduce la pluralidad de descripciones científicas a la determinación de un punto de vista (en cierta manera arbitrario) de la realidad. Esta operación se realiza a partir de la selección y modificación de descripciones conforme el marco de referencia del contexto jurídico, particularmente la argumentación y la referencia - a decisiones precedentes, a parámetros legales, etc.(Trute, 2005, p. 57). Pero esta operación no siempre puede efectuarse. Las controversias científicas se cuelan en el sistema legal, y a menudo trasladan a este sus conflictos.

De modo que, encontrándonos ante casos controversiales, es posible sumar complejidad a la toma de decisiones, atento que el propio campo científico puede tener disidencias respecto a la solución adecuada. Esto representa rediseños en las formas de institucionalidad a la hora de tomar decisiones, y, por lo tanto, oportunidades de participación que el derecho debe procurar accesibles, democráticas y suficientemente controladas.

Estos conflictos científicos, en los cuales se problematiza la solución 'adecuada', según Helmuth Schulze-Fielitz, pueden ser clasificados en tres tipos: conflictos de valores, conflictos sobre los métodos de salvaguarda de los riesgos y conflictos sobre la aplicación de la ciencia y sus implicancias político-sociales-económicas (Schulze-Fielitz, 2005, p. 68). El primer tipo de conflictos, que involucran connotaciones axiológicas en el desarrollo o la implementación de conocimientos científicos - como la clonación o la investigación sobre embriones humanos-, procuran una solución institucionalista. Así, se conforman comités de ética - a menudo integrados por los propios científicos- que deciden acerca de la pertinencia o no de estas investigaciones.

El segundo tipo de conflictos versa sobre la correcta construcción de conocimientos en relación con los riesgos a la salud y otros riesgos en virtud de la implementación de nuevos conocimientos científicos. Estos conflictos han surgido ante la evidencia de que la implementación tecnológica de la ciencia puede acarrear riesgos inesperados, de consecuencias incalculables. Desastres ambientales, atómicos, energéticos y climáticos dan cuenta de las consecuencias no previstas de ciertas aplicaciones 
científicas. ${ }^{39}$ De modo que en los discursos sobre el riesgo se reconocen no solo las voces de los expertos, sino también las de los legos como voces calificadas (Beck, 2008, p. 47), por cuanto la decisión no responde a cálculos científicos, sino a juicios de valor político (Schulze-Fielitz, 2005, p. 68). Ambos autores coinciden en la respuesta jurídica a este problema: mayor institucionalización. En el ámbito del proceso, esta institucionalización puede estar dada por la creación de comisiones de expertos, que establezcan parámetros científicos para evaluar los riesgos.

Finalmente, el tercer tipo de conflicto científico atañe a cuestiones de desarrollo político, social y económico, en las cuales el conocimiento científico es integrado y se vincula con procesos de definición sociopolítico valorativo. En estos casos, el conocimiento científico ejerce un poder de definición sobre riesgos a largo plazo, que se autolegitima. No obstante, puede fundarse tanto en un sustrato racional como en un conocimiento socialmente robusto. Es decir, tanto en uno como en otro caso, las decisiones son políticas y no científicas. Este tipo de conflictos se resuelve también mediante la institucionalización, pero en este caso incluyendo el conocimiento lego. Dado que estos conflictos envuelven problemas políticos, la solución jurídica se ha moldeado a partir de 'consejos asesores' del gobierno, conformados por expertos, la ciudadanía organizada, la industria y los partidos políticos.

En la controversia, las partes también trazan alianzas y diseñan estrategias, trayendo al proceso sus propios asesores técnicos (peritos de parte), citando estudios que obran en revistas científicas, publicaciones que circulan en medios digitales, ofreciendo el testimonio de expertos o personas experimentadas que avalan sus pretensiones, acercando datos recolectados por los propios interesados, etc. Trazar líneas de demarcación entre credibilidad y no credibilidad, en estas ofertas, no es un simple asunto de seguir o aplicar reglas para el juez. Como indica Stehr, no se trata de actos llevados a cabo en una estructura racionalizada, sino que involucran una decisión "personal" (Stehr, 2013, p. 60). La experticia, al contrario de lo que el derecho doctrinariamente presupone, es consti-

39 Es lo que Beck ha denominado "sociedad del riesgo", y que se identifica por una mayor incertidumbre social respecto al desarrollo científico cuyas consecuencias trágicas pueden afectar a todo el planeta (1998, p. 53). 
tuida o reconstituida en cierto grado dentro del marco de cada proceso. Si la manera en que los jueces ven el mundo modela la forma en que otros deben verlo dentro del proceso, entonces la visión judicial debería ser entrenada para reconocer y criticar su propio poder de constreñir las percepciones sociales de la verdad (Jasanoff, 1998, p. 734). ${ }^{40}$

A modo de síntesis de las dimensiones analizadas y los aspectos observables en los casos controversiales, presentamos la tabla 1, en la cual se identifican, así mismo, ciertos espacios en los cuales se escenifica la controversia en el proceso.

Tabla 1. Dimensiones de los 'casos controversiales'

\begin{tabular}{|l|l|l|}
\hline \multicolumn{1}{|c|}{ Dimensión } & \multicolumn{1}{|c|}{ Aspecto observable } & \multicolumn{1}{c|}{ Escenificación de la controversia en el proceso } \\
\hline $\begin{array}{l}\text { Formal/ } \\
\text { estructural }\end{array}$ & $\begin{array}{l}\text { Utilización estratégica } \\
\text { de prueba y experticia } \\
\text { científica. }\end{array}$ & $\begin{array}{l}\text { Coproducción de neutralidad (roles y modos de par- } \\
\text { ticipación en el proceso). }\end{array}$ \\
\hline $\begin{array}{l}\text { Material/ } \\
\text { funcional }\end{array}$ & $\begin{array}{l}\text { Tensiones entre valores } \\
\text { legales y valores } \\
\text { científicos. }\end{array}$ & $\begin{array}{l}\text { Traducción entre imaginarios legales y científicos } \\
\text { (audiencias, gráficos, informes, dictámenes, estanda- } \\
\text { rización). }\end{array}$ \\
\hline $\begin{array}{l}\text { Institucional/ } \\
\text { decisional }\end{array}$ & $\begin{array}{l}\text { Composición judicial } \\
\text { de la solución. }\end{array}$ & $\begin{array}{l}\text { Legitimidad (aceptación o rechazo) de la solución } \\
\text { en la esfera pública (sucesión de leyes en el tiempo, } \\
\text { participación de amicus curiae, conocimientos produ- } \\
\text { cidos por los ciudadanos, comités de gestión). }\end{array}$ \\
\hline
\end{tabular}

Fuente: elaboración de los autores.

La matriz presentada en esta tabla sintetiza las dimensiones analizadas precedentemente. Esta matriz permite identificar y caracterizar los casos controversiales, distinguiéndolos de otros casos complejos. Los aspectos observables en cada una de las dimensiones procuran ofrecer categorías de análisis que permitan individualizar los principales elementos de un caso controversial. A partir de estas categorías, la escenificación de la controversia en el proceso nos brinda los conceptos teóricos para examinar estos casos. De este modo, la matriz configura una herramienta para que los operadores jurídicos puedan abordar el análisis de otros casos controversiales.

40 Denti, por ejemplo, considera que el control del juzgador sobre las nociones tecnocientíficas que se emplean en el proceso debe ser el mismo que puede ejercitar la comunidad (1972, p. 20). 


\section{Conclusiones}

Este artículo propone un recorrido por las notas más salientes de los casos controversiales, a partir de una serie de materiales teóricos provenientes de los ESCT y de la dogmática jurídica procesal y ambiental. Los casos socioambientales presentan un fértil terreno para observar la relación entre ciencia y derecho. Algunos ámbitos, en particular, ofrecen evidencias manifiestas de esta relación, como el tratamiento de la prueba, la participación de expertos y la construcción de la propia decisión judicial. Esto, en el contexto de una sociedad del conocimiento, requiere repensar la epistemología del análisis jurídico.

En este sentido, los casos controversiales problematizan la autoridad de jueces y expertos y renegocian los significados de conceptos tradicionales, como el de 'neutralidad judicial' e 'imparcialidad e independencia del experto'.

El concepto de casos controversiales de este trabajo, construido a partir de la matriz presentada en la tabla 1, da cuenta de la creciente densidad de estos en el universo general de los casos complejos. Es que los casos controversiales son solo una parte de ese universo. Tampoco pueden identificarse exclusivamente con hipótesis de aplicación del principio de precaución, sino que pueden referir a la aplicación del principio de prevención y/o de reparación.

Para conceptualizar los casos controversiales, se ofrece una matriz teórico-práctica que aborda el problema de la incorporación de información científica al proceso, las tensiones que se producen entre valores legales y científicos, y el problema de la aceptación o rechazo de la decisión judicial desde otros ámbitos de participación.

Al aplicar esta matriz al estudio de casos controversiales, resulta que, a diferencia de lo que ocurre en casos tradicionales, se observa que el derecho y la ciencia coproducen neutralidad y negocian el poder de convicción de la prueba desde mundos diversos. Así mismo, la matriz permite visualizar, en el proceso, aquellos conflictos sociales identificados por las ciencias sociales, cuyos valores han sido siempre difíciles de traducir con las reglas clásicas del contradictorio. Finalmente, la matriz enfoca la narrativa de la decisión judicial, y propone una construcción más democrática de su legitimidad. En este sentido, los casos controversiales ofrecen a los 
operadores legales la oportunidad de activar la imaginación institucional que demandan los nuevos conflictos de la sociedad del conocimiento.

Es por estas razones que, al unir los títulos y subtítulos de este trabajo, puede encontrarse la siguiente conclusión: en su aspecto formal, los casos controversiales importan un ejercicio de coproducción de neutralidad, que transita por los cauces del proceso judicial, negociando cientificidad desde mundos diversos; y en su dimensión funcional, revelan profundos conflictos sociales que involucran valores jurídicos y científicos difíciles de traducir con las reglas del contradictorio, trasladando así sus tensiones a instancias decisorias que demandan nuevos arreglos institucionales que permitan la participación de colectivos más amplios, para hacer de la controversia un espacio de oportunidades.

\section{Referencias}

Azuela, A. (2006). Visionarios y pragmáticos: una aproximación sociológica al derecho ambiental. México: Universidad Nacional Autónoma de México.

Bailo, G. (2014). Estrategias jurídicas locales para el gobierno de los nanorresiduos. Construyendo una agenda jurídica regulatoria para nanomateriales en el derecho argentino. Revista Jurídica de Derecho Ambiental, (38), 103-126.

Bailo, G., Bartolini, F., Filippon, C., Levrand, N. E., E Marichal, M. E. (2015). Para muestra basta un botón (o dos): una aproximación a los marcos conceptuales de CTS desde la investigación jurídica. Papeles, (16), 103-121.

Beck, U. (1998). La sociedad del riesgo: hacia una nueva modernidad. Madrid: Paidós Ibérica.

Beck, U. (2008). La sociedad del riesgo mundial: en busca de la seguridad perdida. Madrid: Paidós Ibérica.

Berros, V. (2013). Entramado precautorio. Un aporte desde el derecho para la gestión de riesgos ambientales y relativos a la salud humana en Argentina (Tesis doctoral, Universidad Nacional del Litoral).

Carbone, C. A. (2012). La prueba científica. ¿Valoración por un 'juez de toga blanca'? Revista de Derecho Procesal, 2012(2), 389-430.

Denti, V. (1972). Cientificidad de la prueba y libre valoración del juzgador. Ponencia presentada en el V Congreso Internacional de Derecho Procesal, México. 
Douglas Price, J. (2014). Alcances de la sentencia, criterios de prueba y ejecución de las decisiones en el proceso ambiental. La Ley, Derecho Ambiental, $X X I(1), 1-1$.

Gozaíni, O. (2012). La prueba científica no es prueba pericial. Derecho E Sociedad, 38, 169-175.

Gozaíni, O. (2015). Pruebas científicas y verdad. El mito del razonamiento incuestionable. Recuperado de http://www.derecho.uba.ar/institucional/deinteres/2015gozaini-pruebas-cientificas-y-verdad.pdf

Jasanoff, S. (1998). The eye of everyman witnessing DNA in the Simpson trial. Social Studies of Science, 28(5-6), 713-740.

Jasanoff, S. (2005a). Law's knowledge: science for justice in legal settings. American Journal of Public Health, 95(S1), S49-S58.

Jasanoff, S. (2005b). Civic epistemology. En S. Jasanoff, Designs on nature: science and democracy in Europe and the United States (pp. 247-271). Princeton: Princeton University Press.

Jasanoff, S. (2006). Just evidence: the limits of science in the legal process. The Journal of Law, Medicine E Ethics, 34(2), 328-341.

Jasanoff, S. (2011). Momentos constitucionales en el gobierno de la ciencia y la tecnología. En T. Pérez Bustos E M. Lozano Borda (Eds.), Ciencia, tecnología y democracia: reflexiones en torno a la apropiación social del conocimiento. Medellín: Colciencias-Universidad Eafit.

Jasanoff, S. (2012). Genealogies of STS. Social Studies of Science, 42(3), 435-441. Jasanoff, S. (Ed.). (2004). States of knowledge: the co-production of science and the social order. Routledge.

Jasanoff, S., E Nelkin, D. (1982). Science, technology, and the limits of judicial competence. Jurimetrics, 22(3), 266-278. Recuperado de http://www.jstor. org/stable/29761785

Miller, C. (2004). Climate science and the making of a global political order. En S. Jasanoff, States of knowledge: the co-production of science and the social order (pp. 46-66). Londres: Taylor \& Francis.

Nelkin, D. (1975). The political impact of technical expertise. Social Studies of Science, 5(1), 35-54.

Nelkin, D. (1987). Controversies and the authority of science. En H. Engelhardt $\mathcal{E}$ Arthur L. Caplan (Eds.), Scientific controversies: case studies in the resolution and closure of disputes in science and technology (pp. 283-293). Nueva York: Cambridge University Press.

Nelkin, D. (1995). Science controversies the dynamics of public disputes in the United States. En S. Jasanoff (Ed.), Handbook of science and technology studies. 
Nelkin, D. (Ed.). (1979). Controversy: politics of technical decisions. Beverly Hills: Londres: SAGE.

Nowotny, H. (2011). ¿Salirse de la ciencia es salir de sincronía? En AA. VV. Informe sobre las ciencias sociales en el mundo. Las brechas del conocimiento (pp. 337-340). México: Unesco-Foro Consultivo Científico y Tecnológico.

Peyrano, J. (2007). Sobre la prueba científica. Ius et Veritas, (35), 108-113.

Peyrano, J. (2012). La pericial informativa o pericia colegiada. Revista de Derecho Procesal, (2), 53-62.

Rojas, J. (2012). Reflexiones sobre la 'prueba científica'. Revista de Derecho Procesal, (2), 253-272.

Schulze-Fielitz, H. (2005). Responses of the legal order to the loss of trust in science. En H. Nowotny, D. Pestre, E. Schmidt-Aßmann et al., The public nature of science under assault. politics, markets, science and the law (pp. 63-86). Berlín: Springer.

Shinn, T. (1999). Prefacio. En P. Kreimer, De probetas, computadoras y ratones: la construcción de una mirada sociológica sobre la ciencia. Universidad Nacional de Quilmes.

Stehr, N. (2013). Las sociedades modernas del conocimiento. En: AA. VV. La sociedad de la información y del conocimiento. $N^{0} 11$ (pp. 57-67). Chile: Konrad Adenauer-Stiftung.

Taruffo, M. (2005). Nuevas tendencias del derecho procesal constitucional y legal. Universidad de Medellín.

Taruffo, M., Ibáñez, P. A., Pérez, A. C., E de la Iglesia Monje, M. I. (2009). Consideraciones sobre la prueba judicial. Fundación Coloquio Jurídico Europeo.

Trute, H.-H. (2005). Democratizing science: expertise and participation in administrative decision-making. En H. Nowotny, D. Pestre, E. Schmidt-Aßmann et al., The public nature of science under assault. Politics, markets, science and the law (pp. 87-108). Berlín: Springer.

\section{Jurisprudencia}

Asís María Fernanda c/ Giorda, Mario Luis y otro -ordinario- otros. Cámara Quinta Civil y Comercial, Córdoba. 24/05/2007.

Gioachini, Adolfo vs. YPF S.A. s. Daños y perjuicios. Tercera Cámara de Apelaciones en lo Civil, Comercial, Minas, de Paz y Tributario, Mendoza. 12/08/2015.

Mazzeo, Alicia S. y otro c/ YPF S.A. s. Cese de daño ambiental - daños y perjuicios. Cámara Federal de Apelaciones de La Plata, Sala Tercera. 12/07/2012. 
Mendoza, Beatriz Silvia y otros c/ Estado Nacional y otros s. Daños y perjuicios (daños derivados de la contaminación ambiental del río Matanza-Riachuelo), CSIN, 20/06/2006, fallos: 326:2316.

Peralta, Viviana c/ Municipalidad de San Jorge y otros s. Amparo. Cámara de Apelación en lo Civil y Comercial, Sala Segunda, Santa Fe. 09/12/2009.

Peralta, Viviana y otros c/ Municipalidad de San Jorge y otros s. Amparo. Juzgado de Primera Instancia de Distrito en lo Civil, Comercial y Laboral de San Jorge, Provincia de Santa Fe. 16/03/2009. 